\title{
ON THE 2-RANK AND 4-RANK OF THE CLASS GROUP OF SOME REAL PURE QUARTIC NUMBER FIELDS
}

\author{
MBAREK HAYNOU AND MOHAMMED TAOUS
}

\begin{abstract}
Let $K=\mathbb{Q}\left(\sqrt[4]{p d^{2}}\right)$ be a real pure quartic number field and $k=\mathbb{Q}(\sqrt{p})$ its real quadratic subfield, where $p \equiv 5(\bmod 8)$ is a prime integer and $d$ an odd square-free integer coprime to $p$. In this work, we calculate $r_{2}(K)$, the 2-rank of the class group of $K$, in terms of the number of prime divisors of $d$ that decompose or remain inert in $\mathbb{Q}(\sqrt{p})$, then we will deduce forms of $d$ satisfying $r_{2}(K)=2$. In the last case, the 4-rank of the class group of $K$ is given too.
\end{abstract}

\section{Introduction and Notations}

Let $K$ be a number field and $C l_{2}(K)$ its 2-class group, that is the 2-Sylow subgroup of its class group $C l(K)$. We define the 2-rank and the 4-rank of $C l(K)$ respectively as follows: $r_{2}(K)=\operatorname{dim}_{\mathbb{F}_{2}}\left(C l_{2}(K) / C l_{2}(K)^{2}\right)$ and $r_{4}(K)=\operatorname{dim}_{\mathbb{F}_{2}}\left(C l_{2}(K)^{2} / C l_{2}(K)^{4}\right)$ where $\mathbb{F}_{2}$ is the finite field with 2 elements.

Using genus theory, many mathematicians calculated $r_{2}(K)$ and $r_{4}(K)$ whenever $K$ is a number field having a subfield with odd class number. For instances, we mention the following works:

- For biquadratic number fields, we indicate the works $[1,2,3,4,14,15]$. . .

- For cyclic quartic fields, we indicate the works $[5,6] \ldots$.

- For pure quartic number fields, we indicate the works of Parry [11,12]. . .

In the two last papers, Parry determined the exact power of 2 dividing the class number of some pure quartic number fields.

In this paper, we consider the real pure quartic number fields $K=\mathbb{Q}\left(\sqrt[4]{p d^{2}}\right)=k(\sqrt{d \sqrt{p}})$, where $k=\mathbb{Q}(\sqrt{p})$ with $p \equiv 5(\bmod 8)$ is a prime integer and $d$ is an odd square-free integer satisfying some conditions.

Our first goal is to determine the 2-rank of the class group of $K$, using the ambiguous class number formula in $K / k([7])$ :

$$
\# \mathcal{A} m(K / k)=h(k) \cdot \frac{2^{t-1}}{\left[E_{k}: E_{k} \cap N_{K / k}\left(K^{\times}\right)\right]},
$$

where

- $t$ is the number of finite and infinite primes ramified in $K / k$,

- $E_{k}$ is the unit group of $k$,

- $E_{k} \cap N_{K / k}\left(K^{\times}\right)$is the subgroup of units that are norms of elements of $K$,

- $h(k)$ is the class number of $k$.

2010 Mathematics Subject Classification. 11R11, 11R16, 11R29, 11R37.

Key words and phrases. class groups, pure quartic number field, ambiguous class number formula. 
As $h(k)$ is odd, then the above formula implies that

$$
r_{2}(K)=t-e-1,
$$

with $e$ is an integer defined by $2^{e}=\left[E_{k}: E_{k} \cap N_{K / k}\left(K^{\times}\right)\right]$. Our second goal is to calculate the 4-rank of the class group of fields $K$ satisfying $r_{2}(K)=2$. For this, we will use a formula provided by Y. Qin ([14]). This formula states that the 4-rank of the class group of a quadratic extension $k(\sqrt{\delta})$, where the base field $k$ is of odd class number, is given by:

$$
r_{4}(k(\sqrt{\delta}))=t-1-\operatorname{rank}\left(R_{k(\sqrt{\delta}) / k}\right),
$$

where $t$ is the number of ramified primes in $k(\sqrt{\delta}) / k$ and $R_{k(\sqrt{\delta}) / k}$ is the following matrix:

$$
R_{k(\sqrt{\delta}) / k}=\left(\begin{array}{llll}
\left(\frac{a_{1} ; \delta}{\mathcal{P}_{1}}\right) & \ldots\left(\frac{a_{n} ; \delta}{\mathcal{P}_{1}}\right) & \ldots\left(\frac{a_{n+r} ; \delta}{\mathcal{P}_{1}}\right) \\
\left(\frac{a_{1} ; \delta}{\mathcal{P}_{t}}\right) & \ldots\left(\frac{a_{n} ; \delta}{\mathcal{P}_{t}}\right) & \ldots\left(\frac{a_{n+r} ; \delta}{\mathcal{P}_{t}}\right)
\end{array}\right) .
$$

It is a matrix of type $t \times(n+r)$ with coefficients in $\mathbb{F}_{2}$, called the generalized Rédei-matrix, where $\left(\mathcal{P}_{i}\right)_{1 \leq i \leq t}$ are the primes (finite and infinite) of $k$ which ramify in $k(\sqrt{\delta}),\left(a_{j}\right)_{1 \leq j \leq n+r}$ is a family of elements of $k$ defined by Y. Qin in [14, §2, p. 27] and $\left(\frac{-; \delta}{\mathcal{P}_{i}}\right)$ is the Hilbert symbol on $k$. Note that this matrix is given by replacing the 1's by 0's and the -1's by 1's. For more details concerning the generalized Rédei-matrix, we refer the reader to [14].

During this paper, we adopt the following notations:

- $k=\mathbb{Q}(\sqrt{p})$ with $p \equiv 5(\bmod 8)$ is a prime integer.

- For $z \in k, z^{\prime}$ denotes the conjugate of $z$ over $\mathbb{Q}$.

- $K=\mathbb{Q}\left(\sqrt[4]{p d^{2}}\right)=k(\sqrt{d \sqrt{p}})$, where $d$ is an odd square-free integer such that $(p, d)=1$.

- $\mathcal{O}_{k}$ : the ring of integers of $k$.

- $r_{2}(K)$ : the 2-rank of the class group $C l(K)$.

- $r_{4}(K)$ : the 4-rank of the class group $C l(K)$.

- $\delta=d \sqrt{p}$.

- $\mathcal{P}_{i}$ : a prime ideal of $k$ which ramifies in $K$.

- $N_{K / k}(-)$ : the relative norm of $K$ to $k$.

- $R_{K / k}$ : the generalized Rédei-matrix of $K$.

- $\left(\frac{m}{n}\right)$ : the Legendre symbol.

- $\left[\frac{\overline{\mathcal{P}_{i}}}{\text { - }}\right]$ : the quadratic symbol over $k$.

- $\left(\frac{-,-}{\mathcal{P}_{i}}\right)$ : the Hilbert symbol over $k$.

- $\left(\frac{m}{n}\right)_{4}$ : the rational biquadratic symbol.

- $\varepsilon_{p}$ : the fundamental unit of $k$.

Our main theorems are as follows. Their proofs will be given in Sections 2 and 3.

Theorem A. Suppose that $d=q_{1} \cdots q_{s} q_{1}^{\prime} \cdots q_{t}^{\prime}$ is an odd square-free integer such that $q_{i}$ and $q_{j}^{\prime}$ are distinct primes satisfying $\left(\frac{p}{q_{i}}\right)=-1$ and $\left(\frac{p}{q_{j}^{\prime}}\right)=1$ for each $i, j$. Then the 2 -rank of $C l(K)$ is $r_{2}(K)=2 t+s$. 
Theorem B. If $d=q$ is an odd prime such that $\left(\frac{p}{q}\right)=1$. Then

(1) If $q \equiv 1(\bmod 8)$, then $r_{4}(K)= \begin{cases}0, & \text { if }\left(\frac{p}{q}\right)_{4}=-1, \\ 1, & \text { if }\left(\frac{p}{q}\right)_{4}=-\left(\frac{q}{p}\right)_{4}=1, \\ 2, & \text { if }\left(\frac{p}{q}\right)_{4}=\left(\frac{q}{p}\right)_{4}=1 .\end{cases}$

(2) If $q \equiv 3(\bmod 8)$, then $r_{4}(K)=0$

(3) If $q \equiv 5(\bmod 8)$, then $r_{4}(K)= \begin{cases}0, & \text { if }\left(\frac{p}{q}\right)_{4}=-1, \\ 1, & \text { if }\left(\frac{p}{q}\right)_{4}=1 .\end{cases}$

(4) If $q \equiv 7(\bmod 8)$, then $r_{4}(K)= \begin{cases}0, & \text { if }\left(\frac{q}{p}\right)_{4}=1, \\ 1, & \text { if }\left(\frac{q}{p}\right)_{4}=-1 .\end{cases}$

Theorem C. If $d=q_{1} q_{2}$ where $q_{1}, q_{2}$ are odd prime integers such that $\left(\frac{p}{q_{1}}\right)=\left(\frac{p}{q_{2}}\right)=-1$, then

$$
r_{4}(K)= \begin{cases}1, & \text { if }\left(q_{1}, q_{2}\right) \equiv(3,3) \quad(\bmod 4), \\ 0, & \text { otherwise }\end{cases}
$$

We will analyze the behaviour of this non-Galois extension, $K$, of degree 4 in order to compare, in an other paper in preparation, the results obtained here in this paper with those that can one have when $K$ is replaced by a biquadratic field. The determination of the 2-rank and 4-rank of the class group of $K$, can be also among the most important properties used to study the following problems:

1. Characterize the structure of the 2-group $G=\operatorname{Gal}\left(K_{2}^{(2)} / K\right)$ of $K$ where $K_{2}^{(2)}$ is its second Hilbert 2-class field.

2. Determine the Hilbert 2-class field tower of pure quartic field $K$.

3. Study the capitulation of the 2-ideal classes of the pure quartic field $K$ in the intermediate sub-extensions of $K_{2}^{(1)} / K$ where $K_{2}^{(1)}$ is the first Hilbert 2-class field of $K$.

\section{The 2-rank of $C l(K)$}

In this section, we assume that $d=q_{1} \cdots q_{s} q_{1}^{\prime} \cdots q_{t}^{\prime}$ where $q_{i}$ and $q_{j}^{\prime}$ are distinct primes satisfying $\left(\frac{p}{q_{i}}\right)=-1,\left(\frac{p}{q_{j}^{\prime}}\right)=1$ for each $i, j$.

The relative discriminant of $K / k$ is $\Delta_{K / k}=(4 d \sqrt{p})$ (see [9]), then the finite primes ramified in $K / k$ are $(\sqrt{p}), \tilde{q}_{1}, \cdots, \tilde{q}_{s}, \pi_{1}, \cdots, \pi_{t}, \tilde{\pi}_{1}, \cdots, \tilde{\pi}_{t}$ and $2_{I}$, where $p O_{k}=(\sqrt{p})^{2}, 2_{I}=2 O_{k}$, $\pi_{i} \tilde{\pi}_{i}=q_{i}^{\prime} O_{k}$ and $\tilde{q}_{i}=q_{i} O_{k}$ for each $i$. Regarding infinite primes ramifying in $K / k, k$ has two infinite primes $p_{\infty}$ and $\tilde{p}_{\infty}$ which correspond respectively to the two following $\mathbb{Q}$-embeddings: $i_{p_{\infty}}: \sqrt{p} \longmapsto-\sqrt{p}$ and $i_{\tilde{p}_{\infty}}: \sqrt{p} \longmapsto \sqrt{p}$. As $i_{p_{\infty}}$ can be extend to the two $\mathbb{Q}$-embeddings: $j_{p_{\infty}}: \sqrt{p} \longmapsto i \sqrt[4]{p}$ and $\bar{j}_{p_{\infty}}: \sqrt{p} \longmapsto-i \sqrt[4]{p}$, which are complex embeddings, and $i_{\tilde{p}_{\infty}}$ can be extend to the two $\mathbb{Q}$-embeddings: $j_{\tilde{p}_{\infty}}: \sqrt{p} \longmapsto \sqrt[4]{p}$ and $\bar{j}_{\tilde{p}_{\infty}}: \sqrt{p} \longmapsto-\sqrt[4]{p}$, which are real embeddings, then $p_{\infty}$ is the unique infinite prime of $k$ that ramifies in $K$. 
Lemma 2.1. Keeping previous hypotheses and notations, then

$$
\left(\frac{-1 ; \delta}{p_{\infty}}\right)=\left(\frac{\varepsilon_{p} ; \delta}{\sqrt{p}}\right)=\left(\frac{-\varepsilon_{p} ; \delta}{\sqrt{p}}\right)=-1
$$

Proof.

(1) Let $i_{p_{\infty}}: \sqrt{p} \longmapsto-\sqrt{p}$ be the complex $\mathbb{Q}$-isomorphism of $k$ corresponding to $p_{\infty}$, then by definition of Hilbert symbol given in [8, Ch II $\S 7$ Definitions 7.1, p. 195] and [8, Ch II $\S 7$ Definitions 7.3.1, p. 201], we have,

$$
\left(\frac{-1, \delta}{p_{\infty}}\right)=i_{p_{\infty}}^{-1}\left(\left(i_{p_{\infty}}(-1), i_{p_{\infty}}(\delta)\right)_{p_{\infty}}\right)=i_{p_{\infty}}^{-1}\left((-1,-\delta)_{p_{\infty}}\right)=i_{p_{\infty}}^{-1}(-1)=-1 .
$$

(2) Since $v_{(\sqrt{p})}(\delta)=1$ and via the property [11, Lemma V, p. 105],

$$
\left(\frac{\varepsilon_{p}, \delta}{\sqrt{p}}\right)=\left[\frac{\varepsilon_{p}}{\sqrt{p}}\right]^{v(\delta)}=\left[\frac{\varepsilon_{p}}{\sqrt{p}}\right]=-1 .
$$

(3) We have $\left(\frac{-1, \delta}{\sqrt{p}}\right)=\left[\frac{-1}{\sqrt{p}}\right]=\left(\frac{-1}{p}\right)=1$. Then by applying multiplicative property of Hilbert symbol we get $\left(\frac{-\varepsilon_{p}, \delta}{\sqrt{p}}\right)=\left(\frac{\varepsilon_{p}, \delta}{\sqrt{p}}\right)\left(\frac{-1, \delta}{\sqrt{p}}\right)=-1$.

Proof of Theorem A. Firstly, we know from Hasse norm theorem [8, Ch II $\S 6$, Theorem 6.2, p. 179] that an element $\alpha$ in $k^{\times}$is norm in $K$ if and only if $\left(\frac{\alpha, \delta}{\mathcal{P}_{i}}\right)=1$ for all primes of $k$ ramified in $K$. So, by Lemma (2.1), the units $-1, \varepsilon_{p},-\varepsilon_{p}$ of $k$ are not norms of elements of $K$. Since $E_{k}=\left\langle-1, \varepsilon_{p}\right\rangle$ and $E_{k} \cap N_{K / k}\left(K^{\times}\right)=\left\langle\varepsilon_{p}^{2}\right\rangle$, therefore $\left[E_{k}: E_{k} \cap N_{K / k}\left(K^{\times}\right)\right]=4$, so $e=2$. Secondly, the finite and infinite primes ramified in $K / k$ are $p_{\infty},(\sqrt{p}), 2_{I}, \tilde{q}_{1}, \cdots, \tilde{q}_{s}, \pi_{1}, \cdots, \pi_{t}$, $\tilde{\pi}_{1}, \cdots, \tilde{\pi}_{t}$. Then their number is $2 t+s+3$. Finally, the 2-rank of the class group of $K$ is computed using formula (1).

Corollary 2.2. Keeping previous hypotheses and notations, then $r_{2}(K)=2$ if and only if one of the following conditions holds:

(a) $d=q$ is a prime number such that $\left(\frac{p}{q}\right)=1$,

(b) $d=q_{1} q_{2}$, where $q_{1}, q_{2}$ are odd primes such that $\left(\frac{p}{q_{1}}\right)=\left(\frac{p}{q_{2}}\right)=-1$.

Proof. By Theorem A, $r_{2}(K)=2$ if and only if $(t=1$ and $s=0)$ or $(t=0$ and $s=2)$. Hence,

(a) if $t=1$ and $s=0$, then $d=q$ is a prime number and $\left(\frac{p}{q}\right)=1$,

(b) if $t=0$ and $s=2$, then $d=q_{1} q_{2}$ where $q_{1}, q_{2}$ are odd primes such that $\left(\frac{p}{q_{1}}\right)=\left(\frac{p}{q_{2}}\right)=$ -1 . 


\section{The 4-rank of $C l(K)$}

In this section, we compute the 4-rank of $C l(K)$ whenever $r_{2}(K)=2$. At the end of each case, we give some numerical examples which are verified using the Pari/GP calculator (version 2-11-3), [13].

3.1. Case: $d=q$ is a prime number and $\left(\frac{p}{q}\right)=1$. Let $p$ and $q$ be two different odd prime numbers such that $p \equiv 5(\bmod 8),\left(\frac{p}{q}\right)=1$ and $\pi_{1}, \tilde{\pi}_{1}$ are prime ideals of $\mathcal{O}_{k}$ lying above $q$, the ideals $\pi_{1}^{h}, \tilde{\pi}_{1}^{h}$ are principal ideals of $\mathcal{O}_{k}$, put $\pi_{1}^{h}=(x+y \sqrt{p}) / 2$ and $\tilde{\pi}_{1}^{h}=(x-y \sqrt{p}) / 2$, where $h=h(k)$ is the class number of $k=\mathbb{Q}(\sqrt{p})$. Without loss of generality, we can suppose that $(x-y \sqrt{p})>0$ and $(x+y \sqrt{p})>0$, because

- if $(x-y \sqrt{p})<0$ and $(x+y \sqrt{p})<0$, we replace $(x+y \sqrt{p})$ by $-(x+y \sqrt{p})$ and $(x-y \sqrt{p})$ by $-(x-y \sqrt{p})$. Which are also positive generators for the ideals $\pi_{1}^{h}$ and $\tilde{\pi}_{1}^{h}$ respectively.

- if $(x-y \sqrt{p})>0$ and $(x+y \sqrt{p})<0$, we have $N_{k / \mathbb{Q}}\left(\varepsilon_{p}\right)=\varepsilon_{p} \varepsilon_{p}^{\prime}=-1$ and $\varepsilon_{p}^{\prime}<0$, then we can replace $(x+y \sqrt{p})$ by $\varepsilon_{p}(x+y \sqrt{p})$ and $(x-y \sqrt{p})$ by $\varepsilon_{p}^{\prime}(x-y \sqrt{p})$, which are also positive generators for the ideals $\pi_{1}^{h}, \tilde{\pi}_{1}^{h}$ respectively.

Furthermore, applying absolu norm map to the ideal $\left.\pi_{1}^{h}=(x+y \sqrt{p}) / 2\right)$, one gets $\pm 4 q^{h}=$ $x^{2}-p y^{2}$. But if we considre $x+y \sqrt{p}>0$ and $x-y \sqrt{p}>0$ this equation becomes $4 q^{h}=$ $x^{2}-p y^{2}$. So we can reduce our study to the case when the ideals $\pi_{1}^{h}$ and $\tilde{\pi}_{1}^{h}$ have positive generators.

To compute the 4-rank of the class group of $K$ by using the generalized Rédei-matrix, we need the following lemmas.

Lemma 3.2. Keeping previous hypotheses and notations, then

(a) $\left(\frac{\varepsilon_{p}, \delta}{p_{\infty}}\right)=\left(\frac{\sqrt{p}, \delta}{p_{\infty}}\right)=-1$.

(b) $\left(\frac{2, \delta}{p_{\infty}}\right)=\left(\frac{2(x+y \sqrt{p}), \delta}{p_{\infty}}\right)=\left(\frac{2(x-y \sqrt{p)}, \delta}{p_{\infty}}\right)=1$.

Proof. By the definition of Hilbert symbol given in [8, Ch II, $\S 7$ Definitions 7.1, p. 195] and [8, Ch II $\S 7$ Definitions 7.3.1, p. 201], we have

(a) $\left(\frac{\varepsilon_{p}, \delta}{p_{\infty}}\right)=i_{p_{\infty}}^{-1}\left(\left(i_{p_{\infty}}\left(\varepsilon_{p}\right), i_{p_{\infty}}(\delta)\right)_{p_{\infty}}\right)=i_{p_{\infty}}^{-1}\left(\left(\varepsilon_{p}^{\prime},-\delta\right)_{p_{\infty}}\right)=i_{p_{\infty}}^{-1}(-1)=-1$, since the fundamental unit of $k$ satisfy $\varepsilon_{p}>0$ and $N_{k / \mathbb{Q}}\left(\varepsilon_{p}\right)=\varepsilon_{p} \varepsilon_{p}^{\prime}=-1$, then $\varepsilon_{p}^{\prime}<0$. $\left(\frac{\sqrt{p}, \delta}{p_{\infty}}\right)=i_{p_{\infty}}^{-1}\left(\left(i_{p_{\infty}}(\sqrt{p}), i_{p_{\infty}}(\delta)\right)_{p_{\infty}}\right)=i_{p_{\infty}}^{-1}\left((-\sqrt{p},-\delta)_{p_{\infty}}\right)=i_{p_{\infty}}^{-1}(-1)=-1$.

(b) $\left(\frac{2, \delta}{p_{\infty}}\right)=i_{p_{\infty}}^{-1}\left(\left(i_{p_{\infty}}(2), i_{p_{\infty}}(\delta)\right)_{p_{\infty}}\right)=i_{p_{\infty}}^{-1}\left((2,-\delta)_{p_{\infty}}\right)=i_{p_{\infty}}^{-1}(1)=1$.

$\left(\frac{2(x+y \sqrt{p}), \delta}{p_{\infty}}\right)=i_{p_{\infty}}^{-1}\left(\left(i_{p_{\infty}}(2(x+y \sqrt{p})), i_{p_{\infty}}(\delta)\right)_{p_{\infty}}\right)=i_{p_{\infty}}^{-1}\left((2(x-y \sqrt{p}),-\delta)_{p_{\infty}}\right)=i_{p_{\infty}}^{-1}(1)=1$.

$\left(\frac{2(x-y \sqrt{p}), \delta}{p_{\infty}}\right)=i_{p_{\infty}}^{-1}\left(\left(i_{p_{\infty}}(2(x-y \sqrt{p})), i_{p_{\infty}}(\delta)\right)_{p_{\infty}}\right)=i_{p_{\infty}}^{-1}\left((2(x+y \sqrt{p}),-\delta)_{p_{\infty}}\right)=i_{p_{\infty}}^{-1}(1)=1$. 
Lemma 3.3. Let $p$ and $q$ be distinct odd primes with $p \equiv 1(\bmod 4)$. Then if $(x, y)$ is an integer solution of the equation $u^{2}-p v^{2}=4 q^{h}$, then

$$
\left(\frac{x}{p}\right)=\left(\frac{2}{p}\right)\left(\frac{q}{p}\right)_{4} .
$$

Moreover, if $p \equiv 5(\bmod 8)$, then

$$
\left(\frac{x}{q}\right)= \begin{cases}\left(\frac{p}{q}\right)_{4}, & \text { if } q \equiv 1 \quad(\bmod 4), \\ -\left(\frac{q}{p}\right)_{4}, & \text { if } q \equiv 3 \quad(\bmod 4) .\end{cases}
$$

Proof. Let $(x, y)$ be a solution of the equation $4 q^{h}=u^{2}-p v^{2}$, then $x^{2}$ and $y^{2}$ have same parity, so $x$ and $y$ have same parity too. Hence $4 q^{h} \equiv x^{2}(\bmod p)$. As $p \equiv 1(\bmod 4)$, so

$$
\begin{aligned}
& \left(\frac{x}{p}\right) \equiv\left(x^{2}\right)^{\frac{p-1}{4}} \quad(\bmod p) \\
& \equiv\left(\frac{x^{2}}{p}\right)_{4} \\
& \equiv\left(\frac{4 q^{h}}{p}\right)_{4}^{4} \\
& \equiv\left(\frac{4}{p}\right)_{4}\left(\frac{4}{p}\right)_{4} \\
& =\left(\frac{2}{p}\right)^{4}\left(\frac{q}{p}\right)_{4} \text {. }
\end{aligned}
$$

For the second equality, put $x=2^{r} x^{\prime}$ and $y=2^{e} y^{\prime}$ such that $2 \nmid x^{\prime}$ and $2 \nmid y^{\prime}$.

(a) If $q \equiv 1(\bmod 4)$, then $\left(\frac{x}{q}\right)=\left(\frac{x^{2}}{q}\right)_{4}=\left(\frac{y^{2} p}{q}\right)$

$$
\begin{aligned}
& =\left(\frac{y}{q}\right)^{e}\left(\frac{p}{q}\right)_{4}=\left(\frac{2}{q}\right)^{e}\left(\frac{q}{y^{\prime}}\right)\left(\frac{p}{q}\right)_{4} \\
& =\left(\frac{2}{q}\right)^{e}\left(\frac{4 q^{h}}{y^{\prime}}\right)\left(\frac{p}{q}\right)_{4} \\
& =\left(\frac{2}{q}\right)^{e}\left(\frac{p}{q}\right)_{4} .
\end{aligned}
$$

- If $q \equiv 1(\bmod 8)$ or $y$ is odd, then $\left(\frac{x}{q}\right)=\left(\frac{p}{q}\right)_{4}$.

- If $q \equiv 5(\bmod 8)$ and $x, y$ are even, then $x=2 a$ and $y=2 b$, so $q^{h}=a^{2}-p b^{2}$. Hence by calculation modulo 8 and taking into account the fact that $h$ is odd, we get the following table:

\begin{tabular}{c|cccccccc}
\hline \hline$a$ & 0 & 1 & 2 & 3 & 4 & 5 & 6 & 7 \\
\hline$a^{2}$ & 0 & 1 & 4 & 1 & 0 & 1 & 4 & 1 \\
\hline $5-a^{2}$ & 5 & 4 & 1 & 4 & 5 & 4 & 1 & 4 \\
\hline
\end{tabular}

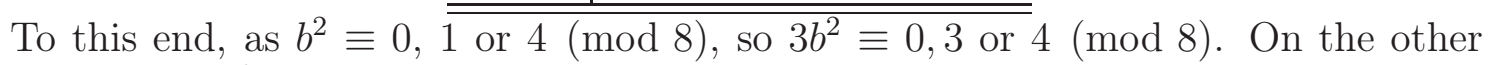
hand, since $q^{h}-a^{2} \equiv 5-a^{2} \equiv 3 b^{2}(\bmod 8)$ one deduces that $3 b^{2} \equiv 4(\bmod 8)$, thus multiplying by the inverse of $3(\bmod 8)$ we get $b^{2} \equiv 4(\bmod 8)$. Hence $e=2$, this in turn yields that

$$
\left(\frac{x}{q}\right)=\left(\frac{p}{q}\right)_{4} .
$$


(b) If $q \equiv 3(\bmod 4)$, then by quadratic reciprocity low we get

$$
\left(\frac{x}{q}\right)=\left(\frac{2^{r} x^{\prime}}{q}\right)=\left(\frac{2}{q}\right)^{r}\left(\frac{-q}{x^{\prime}}\right)=\left(\frac{2}{q}\right)^{r}\left(\frac{-4 q^{h}}{x^{\prime}}\right)=\left(\frac{2}{q}\right)^{r}\left(\frac{p}{x^{\prime}}\right)=\left(\frac{2}{q}\right)^{r}\left(\frac{2}{p}\right)^{r}\left(\frac{x}{p}\right)=\left(\frac{2}{q}\right)^{r}\left(\frac{q}{p}\right)_{4}(-1)^{r+1} .
$$

- If $q \equiv 3(\bmod 8)$ or $x$ is odd, then $\left(\frac{x}{q}\right)=-\left(\frac{q}{p}\right)_{4}$.

- If $q \equiv 7(\bmod 8)$ and $x$ and $y$ are even $x=2 a$ and $y=2 b$, so $q^{h}=a^{2}-p b^{2}$, hence by calculation modulo 8 we get the following table:

\begin{tabular}{c|cccccccc}
\hline \hline$b$ & 0 & 1 & 2 & 3 & 4 & 5 & 6 & 7 \\
\hline$b^{2}$ & 0 & 1 & 4 & 1 & 0 & 1 & 4 & 1 \\
\hline $7+5 b^{2}$ & 7 & 4 & 3 & 4 & 7 & 4 & 3 & 4 \\
\hline \hline
\end{tabular}

with the following congruences : $b^{2} \equiv 0,1$ or $4(\bmod 8)$ and $a^{2} \equiv q^{h}+p b^{2} \equiv$ $7+5 b^{2}$,we find that $a^{2} \equiv 4(\bmod 8)$, then $r=2$. Which also means that

$$
\left(\frac{x}{q}\right)=-\left(\frac{q}{p}\right)_{4} \text {. }
$$

Lemma 3.4. Let $p$ and $q$ be two different odd prime numbers such that $p \equiv 5(\bmod 8)$ and $\left(\frac{p}{q}\right)=1$, then

(a) $\left(\frac{2, \delta}{\sqrt{p}}\right)=-1$.

(b) $\left(\frac{\sqrt{p}, \delta}{\sqrt{p}}\right)=-\left(\frac{\varepsilon_{p}, \delta}{\sqrt{p}}\right)=1$.

(c) $\left(\frac{2(x+y \sqrt{p}), \delta}{\sqrt{p}}\right)=\left(\frac{2(x-y \sqrt{p}), \delta}{\sqrt{p}}\right)=\left(\frac{q}{p}\right)_{4}$.

Proof. We have

(a) $\left(\frac{2, \delta}{\sqrt{p}}\right)=\left[\frac{2}{\sqrt{p}}\right]=\left(\frac{2}{p}\right)=-1$.

(b) $\left(\frac{\sqrt{p}, \delta}{\sqrt{p}}\right)=\left[\frac{-q}{\sqrt{p}}\right]=\left(\frac{-1}{p}\right)\left(\frac{q}{p}\right)=\left(\frac{p}{q}\right)=1$.

(c) $\left(\frac{2(x+y \sqrt{p}), \delta}{\sqrt{p}}\right)=\left[\frac{2(x+y \sqrt{p})}{\sqrt{p}}\right]=\left(\frac{2 x}{p}\right)=\left(\frac{2}{p}\right)\left(\frac{x}{p}\right)=\left(\frac{q}{p}\right)_{4}$. A similar argument shows that $\left(\frac{2(x-y \sqrt{p}), \delta}{\sqrt{p}}\right)=\left(\frac{q}{p}\right)_{4}$.

Lemma 3.5. Keep hypotheses and notations of Lemma 3.4, then

(a) $\left(\frac{-1, \delta}{\pi_{1}}\right)=\left(\frac{-1, \delta}{\pi_{1}}\right)= \begin{cases}1, & \text { if } q \equiv 1(\bmod 4), \\ -1, & \text { if } q \equiv 3(\bmod 4) .\end{cases}$

(b) - If $q \equiv 1(\bmod 4)$, then $\left(\frac{\varepsilon_{p}, \delta}{\pi_{1}}\right)=\left(\frac{\varepsilon_{p}, \delta}{\tilde{\pi}_{1}}\right)=\left(\frac{p}{q}\right)_{4}\left(\frac{q}{p}\right)_{4}$.

- If $q \equiv 3(\bmod 4)$, then $\left(\frac{\varepsilon_{p}, \delta}{\pi_{1}}\right)=-\left(\frac{\varepsilon_{p}, \delta}{\pi_{1}}\right)=-\left(\frac{y}{q}\right)$.

(c) $\left(\frac{2, \delta}{\pi_{1}}\right)=\left(\frac{2, \delta}{\tilde{\pi}_{1}}\right)=\left(\frac{2}{q}\right)$. 
(d)

- If $q \equiv 1(\bmod 4)$, then $\left(\frac{\sqrt{p}, \delta}{\pi_{1}}\right)=\left(\frac{\sqrt{p}, \delta}{\tilde{\pi}_{1}}\right)=\left(\frac{p}{q}\right)_{4}$.

- If $q \equiv 3(\bmod 4)$, then $\left(\frac{\sqrt{p}, \delta}{\pi_{1}}\right)=-\left(\frac{\sqrt{p}, \delta}{\tilde{\pi}_{1}}\right)=\left(\frac{y}{q}\right)\left(\frac{q}{p}\right)_{4}$.

(e) - If $q \equiv 1(\bmod 4)$, then $\left(\frac{2(x+y \sqrt{p}), \delta}{\pi_{1}}\right)=\left(\frac{2(x-y \sqrt{p}), \delta}{\tilde{\pi}_{1}}\right)=1$.

- If $q \equiv 3(\bmod 4)$, then $\left(\frac{2(x+y \sqrt{p}), \delta}{\pi_{1}}\right)=-\left(\frac{2(x-y \sqrt{p}), \delta}{\tilde{\pi}_{1}}\right)=\left(\frac{y}{q}\right)$.

(f) $\left(\frac{2(x-y \sqrt{p}), \delta}{\pi_{1}}\right)=\left(\frac{2(x+y \sqrt{p}), \delta}{\tilde{\pi}_{1}}\right)= \begin{cases}\left(\frac{p}{q}\right)_{4}, & \text { if } q \equiv 1(\bmod 4), \\ -\left(\frac{q}{p}\right)_{4}, & \text { if } q \equiv 3(\bmod 4) .\end{cases}$

Proof. The main tools to prove these equalities are the properties cited in $[8, \mathrm{Ch}$ II $\S 7$ Proposition 7.4.3, p. 205] and by applying the bilinear property of Hilbert symbol, we get

(a) $\left(\frac{-1, \delta}{\pi_{1}}\right)=\left(\frac{-1, \delta}{\pi_{1}}\right)=\left[\frac{-1}{\pi_{1}}\right]=\left(\frac{-1}{q}\right)= \begin{cases}1, & \text { if } q \equiv 1(\bmod 4), \\ -1, & \text { if } q \equiv 3(\bmod 4) \text {. }\end{cases}$

(b) $\bullet$ Suppose $q \equiv 1(\bmod 4)$. Since $q \equiv p \equiv 1(\bmod 4)$ and $\left(\frac{p}{q}\right)=1$, then by Scholz's reciprocity law ( $[10$, Ch $5 \S 5.2$ Proposition 5.8, p. 160]), we have

$\left(\frac{\varepsilon_{p}, \delta}{\pi_{1}}\right)=\left[\frac{\varepsilon_{p}}{\pi_{1}}\right]=\left(\frac{p}{q}\right)_{4}\left(\frac{q}{p}\right)_{4}$, moreover $\left[\frac{\varepsilon_{p}}{\pi_{1}}\right]\left[\frac{\varepsilon_{p}}{\tilde{\pi}_{1}}\right]=\left(\frac{-1}{q}\right)=1$, then $\left(\frac{\varepsilon_{p}, \delta}{\pi_{1}}\right)=$ $\left(\frac{p}{q}\right)_{4}\left(\frac{q}{p}\right)_{4}$.

- If $q \equiv 3(\bmod 4)$ we find that $\left(\frac{\varepsilon_{p}, \delta}{\pi_{1}}\right)=\left[\frac{\varepsilon_{p}}{\pi_{1}}\right]$ and $\left(\frac{\varepsilon_{p}, \delta}{\tilde{\pi}_{1}}\right)=\left[\frac{\varepsilon_{p}}{\tilde{\pi}_{1}}\right]$.

Consequently, $\left[\frac{\varepsilon_{p}}{\pi_{1}}\right]\left[\frac{\varepsilon_{p}}{\tilde{\pi_{1}}}\right]=\left[\frac{\varepsilon_{p}}{\tilde{\pi_{1}} \pi_{1}}\right]=\left[\frac{\varepsilon_{p}}{q O_{k}}\right]=\left(\frac{N_{k / \mathbb{Q}}\left(\varepsilon_{p}\right)}{q}\right)=\left(\frac{-1}{q}\right)=-1$.

As $\left[\frac{\varepsilon_{p} \sqrt{p}}{\pi_{1}}\right]=-\left(\frac{q}{p}\right)_{4}$ (via [16]) and $\left[\frac{\varepsilon_{p} \sqrt{p}}{\pi_{1}}\right]=\left[\frac{-\varepsilon_{p}}{\pi_{1}}\right]\left[\frac{-\sqrt{p}}{\pi_{1}}\right]=\left(\frac{-1}{q}\right)\left[\frac{\varepsilon_{p}}{\pi_{1}}\right]\left(\frac{y}{q}\right)\left(\frac{2}{p}\right)\left(\frac{q}{p}\right)_{4}$.

Then $\left[\frac{\varepsilon_{p}}{\pi_{1}}\right]=-\left(\frac{-1}{q}\right)\left(\frac{2}{p}\right)\left(\frac{y}{q}\right)=\left(\frac{2}{p}\right)\left(\frac{y}{q}\right)$. This completes the proof.

(c) $\left(\frac{2, \delta}{\pi_{1}}\right)=\left[\frac{2}{\pi_{1}}\right]=\left(\frac{2}{q}\right)$ and $\left(\frac{2, \delta}{\pi_{1}}\right)=\left[\frac{2}{\tilde{\pi}_{1}}\right]=\left(\frac{2}{q}\right)$.

(d) - If $q \equiv 1(\bmod 4)$, then

$\left(\frac{\sqrt{p}, \delta}{\pi_{1}}\right)=\left[\frac{\sqrt{p}}{\pi_{1}^{h}}\right]=\left[\frac{2 y}{\pi_{1}^{h}}\right]\left[\frac{2 x-2(x+y \sqrt{p})}{\pi_{1}^{h}}\right]=\left[\frac{2 y}{\pi_{1}}\right]\left[\frac{2 x}{\pi_{1}}\right]=\left(\frac{y}{q}\right)\left(\frac{x}{q}\right)=\left(\frac{p}{q}\right)_{4}$,

$\left(\frac{\sqrt{p}, \delta}{\tilde{\pi_{1}}}\right)=\left[\frac{\sqrt{p}}{\tilde{\pi}_{1}{ }^{h}}\right]=\left[\frac{2 y}{\tilde{\pi}_{1}}\right]\left[\frac{-2 x+2(x-y \sqrt{p})}{\tilde{\pi_{1}}{ }^{h}}\right]=\left[\frac{2 y}{\tilde{\pi_{1}}}\right]\left[\frac{-2 x}{\tilde{\pi_{1}}}\right]=\left(\frac{y}{q}\right)\left(\frac{-1}{q}\right)\left(\frac{x}{q}\right)=\left(\frac{p}{q}\right)_{4}$.

- In the same way, we show that if $q \equiv 3(\bmod 4)$, then

$$
\begin{aligned}
& \left(\frac{\sqrt{p}, \delta}{\pi_{1}}\right)=\left[\frac{\sqrt{p}}{\pi_{1}^{h}}\right]=\left[\frac{2 y}{\pi_{1}^{h}}\right]\left[\frac{2 y \sqrt{p}}{\pi_{1}^{h}}\right]=\left[\frac{2 y}{\pi_{1}}\right]\left[\frac{-2 x}{\tilde{\pi_{1}}}\right]=\left(\frac{-1}{q}\right)\left(\frac{y}{q}\right)\left(\frac{x}{q}\right)=\left(\frac{y}{q}\right)\left(\frac{q}{p}\right)_{4}, \\
& \left(\frac{\sqrt{p}, \delta}{\tilde{\pi_{1}}}\right)=\left[\frac{\sqrt{p}}{\tilde{\pi_{1}}}\right]=\left[\frac{2 y}{\tilde{\pi_{1}}{ }^{h}}\right]\left[\frac{2 y \sqrt{p}}{\tilde{\pi_{1}}}\right]=\left[\frac{2 y}{\tilde{\pi_{1}}}\right]\left[\frac{2 x}{\tilde{\pi_{1}}}\right]=\left(\frac{y}{q}\right)\left(\frac{x}{q}\right)=-\left(\frac{y}{q}\right)\left(\frac{q}{p}\right)_{4} .
\end{aligned}
$$


(e) We also have that if $q \equiv 1(\bmod 4)$, then

$$
\begin{aligned}
& \left(\frac{2(x+y \sqrt{p}), \delta}{\pi_{1}}\right)=\left(\frac{2(x+y \sqrt{p}), \delta}{\pi_{1}}\right)\left(\frac{-\sqrt{p}(x-y \sqrt{p}), \delta}{\pi_{1}}\right)\left(\frac{-\sqrt{p}(x-y \sqrt{p}), \delta}{\pi_{1}}\right) \\
& =\left(\frac{-8 q^{h} \sqrt{p}, \delta}{\pi_{1}}\right)\left(\frac{-\sqrt{p}(x-y \sqrt{p}), \delta}{\pi_{1}}\right) \\
& =\left(\frac{-\delta, \delta}{\pi_{1}}\right)\left(\frac{-2 \sqrt{p}(x-y \sqrt{p}), \delta}{\pi_{1}}\right)=\left[\frac{-2 \sqrt{p}(x-y \sqrt{p})}{\pi_{1}}\right] \\
& =\left[\frac{-2 \sqrt{p}(x-y \sqrt{p})}{\pi_{1}^{h}}\right]=\left[\frac{-\sqrt{p}}{\pi_{1}^{h}}\right]\left[\frac{2(x-y \sqrt{p})}{\pi_{1}^{h}}\right] \\
& =\left[\frac{-\sqrt{p}}{\pi_{1}^{h}}\right]\left[\frac{4 x-2(x+y \sqrt{p})}{\pi_{1}^{h}}\right]=\left[\frac{-\sqrt{p}}{\pi_{1}^{h}}\right]\left[\frac{4 x}{\pi_{1}^{h}}\right] \\
& =\left[\frac{-\sqrt{p}}{\pi_{1}^{h}}\right]\left[\frac{x}{\pi_{1}}\right]=\left(\frac{p}{q}\right)_{4}\left(\frac{x}{q}\right)^{=}=\left(\frac{p}{q}\right)_{4}\left(\frac{p}{q}\right)_{4}=1 . \\
& \left(\frac{2(x-y \sqrt{p}), \delta}{\tilde{\pi}_{1}}\right)=\left(\frac{2(x-y \sqrt{p}), \delta}{\tilde{\pi}_{1}}\right)\left(\frac{-\sqrt{p}(x+y \sqrt{p}), \delta}{\tilde{\pi}_{1}}\right)\left(\frac{-\sqrt{p}(x+y \sqrt{p}), \delta}{\tilde{\pi}_{1}}\right) \\
& =\left(\frac{-\delta, \delta}{\tilde{\pi}_{1}}\right)\left(\frac{-2 \sqrt{p}(x+y \sqrt{p}), \delta}{\tilde{\pi}_{1}}\right)=\left[\frac{-2 \sqrt{p}(x+y \sqrt{p})}{\tilde{\pi}_{1}}\right] \\
& =\left[\frac{-\sqrt{p}}{\tilde{\pi}_{1}}\right]\left[\frac{2(x+y \sqrt{p})}{\tilde{\pi_{1}}}\right]=\left[\frac{-\sqrt{p}}{\tilde{\pi}_{1}^{h}}\right]\left[\frac{2(x+y \sqrt{p})}{\tilde{\pi}_{1}^{h}}\right]=\left[\frac{-\sqrt{p}}{\tilde{\pi}_{1}^{h}}\right]\left[\frac{4 x-2(x-y \sqrt{p})}{\tilde{\pi}_{1}^{h}}\right] \\
& =\left[\frac{-\sqrt{p}}{\tilde{\pi_{1}}{ }^{h}}\right]\left[\frac{4 x}{\tilde{\pi_{1}} h}\right]=\left[\frac{-\sqrt{p}}{\tilde{\pi_{1}} h}\right]\left[\frac{x}{\tilde{\pi_{1}}}\right] \\
& =\left(\frac{p}{q}\right)_{4}\left(\frac{x}{q}\right)=\left(\frac{p}{q}\right)_{4}\left(\frac{p}{q}\right)_{4}=1 .
\end{aligned}
$$

- If $q \equiv 3(\bmod 4)$, then

$$
\begin{aligned}
& \left(\frac{2(x+y \sqrt{p}), \delta}{\pi_{1}}\right)=\left(\frac{2(x+y \sqrt{p}), \delta}{\pi_{1}}\right)\left(\frac{-2 \sqrt{p}(x-y \sqrt{p}), \delta}{\pi_{1}}\right)\left(\frac{-2 \sqrt{p}(x-y \sqrt{p}), \delta}{\pi_{1}}\right) \\
& =\left(\frac{-4 q^{h} \sqrt{p}, \delta}{\pi_{1}}\right)\left(\frac{-2 \sqrt{p}(x-y \sqrt{p}), \delta}{\pi_{1}}\right) \\
& =\left(\frac{-\delta, \delta}{\pi_{1}}\right)\left(\frac{-2 \sqrt{p}(x-y \sqrt{p}), \delta}{\pi_{1}}\right) \\
& =\left[\frac{-2 \sqrt{p}(x-y \sqrt{p})}{\pi_{1}}\right]=\left[\frac{-2 \sqrt{p}(x-y \sqrt{p})}{\pi_{1}^{h}}\right] \\
& =\left[\frac{-\sqrt{p}}{\pi_{1}^{h}}\right]\left[\frac{2(x-y \sqrt{p})}{\pi_{1}^{h}}\right]=\left[\frac{-\sqrt{p}}{\pi_{1}^{h}}\right]\left[\frac{4 x-2(x+y \sqrt{p})}{\pi_{1}^{h}}\right] \\
& =\left[\frac{-\sqrt{p}}{\pi_{1}^{h}}\right]\left[\frac{4 x}{\pi_{1}^{h}}\right]=\left[\frac{-\sqrt{p}}{\pi_{1}^{h}}\right]\left[\frac{x}{\pi_{1}}\right] \\
& =\left(\frac{y}{q}\right)\left(\frac{x}{q}\right)\left(\frac{x}{q}\right)=\left(\frac{y}{q}\right) \text {. } \\
& \left(\frac{2(x-y \sqrt{p}), \delta}{\tilde{\pi}_{1}}\right)=\left(\frac{2(x-y \sqrt{p}), \delta}{\tilde{\pi_{1}}}\right)\left(\frac{-2 \sqrt{p}(x+y \sqrt{p}), \delta}{\tilde{\pi}_{1}}\right)\left(\frac{-2 \sqrt{p}(x+y \sqrt{p}), \delta}{\tilde{\pi}_{1}}\right) \\
& =\left(\frac{-\delta, \delta}{\tilde{\pi}_{1}}\right)\left(\frac{-2 \sqrt{p}(x+y \sqrt{p}), \delta}{\tilde{\pi_{1}}}\right)=\left[\frac{-2 \sqrt{p}(x+y \sqrt{p})}{\tilde{\pi_{1}}}\right] \\
& =\left[\frac{-\sqrt{p}}{\tilde{\pi_{1}}}\right]\left[\frac{2(x+y \sqrt{p})}{\tilde{\pi_{1}}}\right]=\left[\frac{-\sqrt{p}}{\tilde{\pi}_{1}^{h}}\right]\left[\frac{2(x+y \sqrt{p})}{\tilde{\pi}_{1}^{h}}\right] \\
& =\left[\frac{x}{\tilde{\pi}_{1}{ }^{h}}\right]\left[\frac{4 x-2(x-y \sqrt{p})}{\tilde{\pi}_{1}{ }^{h}}\right]=\left[\frac{-\sqrt{p}}{\tilde{\pi}_{1}{ }^{h}}\right]\left[\frac{4 x}{\tilde{\pi}_{1}{ }^{h}}\right] \\
& =\left[\frac{-\sqrt{p}}{\tilde{\pi_{1}^{h}}}\right]\left[\frac{x}{\tilde{\pi_{1}}}\right]=-\left(\frac{y}{q}\right)\left(\frac{x}{q}\right)\left(\frac{x}{q}\right)=-\left(\frac{y}{q}\right) .
\end{aligned}
$$

(f) With similar calculations we prove that

- If $q \equiv 1(\bmod 4)$, then

$$
\begin{aligned}
& \left(\frac{2(x+y \sqrt{p}), \delta}{\tilde{\pi_{1}}}\right)=\left[\frac{2(x+y \sqrt{p})}{\tilde{\pi}_{1}}\right]=\left[\frac{4 x}{\tilde{\pi_{1}}}\right]=\left(\frac{x}{q}\right)=\left(\frac{p}{q}\right)_{4}, \\
& \text { and } \\
& \left(\frac{2(x-y \sqrt{p}), \delta}{\pi_{1}}\right)=\left[\frac{2(x-y \sqrt{p})}{\pi_{1}}\right]=\left[\frac{4 x}{\pi_{1}}\right]=\left(\frac{x}{q}\right)=\left(\frac{p}{q}\right)_{4} .
\end{aligned}
$$


- If $q \equiv 3(\bmod 4)$, then

$$
\begin{aligned}
& \left(\frac{2(x+y \sqrt{p}), \delta}{\tilde{\pi_{1}}}\right)=\left[\frac{2(x+y \sqrt{p})}{\tilde{\pi_{1}}}\right]=\left[\frac{4 x}{\tilde{\pi}_{1}}\right]=\left(\frac{x}{q}\right)=\left(\frac{2}{p}\right)\left(\frac{q}{p}\right)_{4}=-\left(\frac{q}{p}\right)_{4}, \\
& \text { and }\left(\frac{2(x-y \sqrt{p}), \delta}{\pi_{1}}\right)=\left[\frac{2(x-y \sqrt{p})}{\pi_{1}}\right]=\left[\frac{4 x}{\pi_{1}}\right]=\left(\frac{x}{q}\right)=\left(\frac{2}{p}\right)\left(\frac{q}{p}\right)_{4}=-\left(\frac{q}{p}\right)_{4} .
\end{aligned}
$$

Lemma 3.6. Keeping previous hypotheses and notations, then

(a) $\left(\frac{\varepsilon_{p}, \delta}{2_{I}}\right)= \begin{cases}1, & \text { if } q \equiv 1(\bmod 4), \\ -1, & \text { if } q \equiv 3(\bmod 4) .\end{cases}$

(b) $\left(\frac{2, \delta}{2_{I}}\right)=\left(\frac{-1, \delta}{2_{I}}\right)=-1$, and $\left(\frac{\sqrt{p}, \delta}{2_{I}}\right)= \begin{cases}-1, & \text { if } q \equiv 1(\bmod 4) \text {, } \\ 1, & \text { if } q \equiv 3(\bmod 4) \text {. }\end{cases}$

(c) - If $q \equiv 1(\bmod 4)$, then $\left(\frac{2(x+y \sqrt{p}), \delta}{2_{I}}\right)=\left(\frac{2(x-y \sqrt{p}), \delta}{2_{I}}\right)=\left(\frac{p}{q}\right)_{4}\left(\frac{q}{p}\right)_{4}$.

- If $q \equiv 3(\bmod 4)$, then $\left(\frac{2(x+y \sqrt{p}), \delta}{2_{I}}\right)=-\left(\frac{2(x-y \sqrt{p}), \delta}{2_{I}}\right)=-\left(\frac{y}{q}\right)$.

Proof. We prove these results by using the product formula for Hilbert symbol and previous lemmas.

Remark 3.7. The above results can be summered in the following tables. For $q \equiv 1$ $(\bmod 4)$, we have

\begin{tabular}{||c||c|c|c|c|c||}
\hline \hline element & $\pi_{1}$ & $\tilde{\pi}_{1}$ & $2_{I}$ & $(\sqrt{p})$ & $p_{\infty}$ \\
\hline \hline $2(x+y \sqrt{p})$ & 1 & $\left(\frac{p}{q}\right)_{4}$ & $\left(\frac{p}{q}\right)_{4}\left(\frac{q}{p}\right)_{4}$ & $\left(\frac{q}{p}\right)_{4}$ & 1 \\
\hline $2(x-y \sqrt{p})$ & $\left(\frac{p}{q}\right)_{4}$ & 1 & $\left(\frac{p}{q}\right)_{4}\left(\frac{q}{p}\right)_{4}$ & $\left(\frac{q}{p}\right)_{4}$ & 1 \\
\hline 2 & $\left(\frac{2}{q}\right)_{1}$ & $\left(\frac{2}{q}\right)$ & -1 & -1 & 1 \\
\hline$\sqrt{p}$ & $\left(\frac{p}{q}\right)_{4}$ & $\left(\frac{p}{q}\right)_{4}$ & -1 & 1 & -1 \\
\hline-1 & 1 & 1 & -1 & 1 & -1 \\
\hline$\varepsilon_{p}$ & $\left(\frac{p}{q}\right)_{4}\left(\frac{q}{p}\right)_{4}$ & $\left(\frac{p}{q}\right)_{4}\left(\frac{q}{p}\right)_{4}$ & 1 & -1 & -1 \\
\hline \hline
\end{tabular}

For $q \equiv 3(\bmod 4)$, we have $\left(p^{\frac{(q+1)}{8}}\right)^{4} \equiv p^{\frac{(q+1)}{2}} \equiv p^{\frac{(q-1+2)}{2}} \equiv p^{\frac{(q-1)}{2}+1} \equiv\left(\frac{p}{q}\right) p \equiv p(\bmod q)$, then $p^{\frac{(q+1)}{8}}$ is a solution to $x^{4} \equiv p(\bmod q)$, hence $\left(\frac{p}{q}\right)_{4}=1$. 


\begin{tabular}{||c||c|c|c|c|c||}
\hline \hline element & $\pi_{1}$ & $\tilde{\pi}_{1}$ & $2_{I}$ & $(\sqrt{p})$ & $p_{\infty}$ \\
\hline \hline $2(x+y \sqrt{p})$ & $\left(\frac{y}{q}\right)$ & $-\left(\frac{q}{p}\right)_{4}$ & $-\left(\frac{y}{q}\right)$ & $\left(\frac{q}{p}\right)_{4}$ & 1 \\
\hline $2(x-y \sqrt{p})$ & $-\left(\frac{q}{p}\right)_{4}$ & $-\left(\frac{y}{q}\right)$ & $\left(\frac{y}{q}\right)$ & $\left(\frac{q}{p}\right)_{4}$ & 1 \\
\hline 2 & $\left(\frac{2}{q}\right)$ & $\left(\frac{2}{q}\right)$ & -1 & -1 & 1 \\
\hline$\sqrt{p}$ & $\left(\frac{y}{q}\right)^{\left(\frac{q}{p}\right)_{4}}$ & $-\left(\frac{y}{q}\right)\left(\frac{q}{p}\right)_{4}$ & 1 & 1 & -1 \\
\hline-1 & -1 & -1 & -1 & 1 & -1 \\
\hline$\varepsilon_{p}$ & $-\left(\frac{y}{q}\right)$ & $\left(\frac{y}{q}\right)$ & -1 & -1 & -1 \\
\hline \hline
\end{tabular}

\section{Proof of Theorem B.}

By assuming $q$ is a prime number satisfying $\left(\frac{p}{q}\right)=1$, the finite primes of $k$ ramifying in $K$ are: $\pi_{1}, \tilde{\pi}_{1}, 2_{I}$ and $(\sqrt{p})$. So there exist prime ideals $\mathcal{H}_{1}, \mathcal{H}_{2}, \mathcal{H}_{3}, \mathcal{H}_{4}$ of $K$ such that

$$
\mathcal{H}_{1}^{2}=\pi_{1} O_{K}, \mathcal{H}_{2}^{2}=\tilde{\pi}_{1} O_{K}, \mathcal{H}_{3}^{2}=2_{I} O_{K}, \mathcal{H}_{4}^{2}=\sqrt{p} O_{K} .
$$

Therefore, we have

- $\mathcal{H}_{1}^{h} \sigma\left(\mathcal{H}_{1}^{h}\right)=\mathcal{H}_{1}^{h} \mathcal{H}_{1}^{h} O_{K}=\pi_{1}^{h} O_{K}=((x+y \sqrt{p}) / 2) O_{K}$,

- $\mathcal{H}_{2}^{h} \sigma\left(\mathcal{H}_{2}^{h}\right)=\mathcal{H}_{2}^{h} \mathcal{H}_{2}^{h} O_{K}=\tilde{\pi}_{1}^{h} O_{K}=((x-y \sqrt{p}) / 2) O_{K}$,

- $\mathcal{H}_{3} \sigma\left(\mathcal{H}_{3}\right)=\mathcal{H}_{3} \mathcal{H}_{3} O_{K}=2_{I} O_{K}=2 O_{K}$,

- $\mathcal{H}_{4} \sigma\left(\mathcal{H}_{4}\right)=\mathcal{H}_{4} \mathcal{H}_{4} O_{K}=\sqrt{p} O_{K}=\sqrt{p} O_{K}$.

where $\sigma$ is the generator of the Galois group of $K / k$, and $h$ the class number of $k$.

Moreover, we know from Lemma 2.1 that $-1, \varepsilon_{p},-\varepsilon_{p} \notin E_{k} \cap N_{K / k}(K)$. Then the $5 \times(4+2)$ generalized Rédei's matrix of Hilbert symbols (3), can be written as

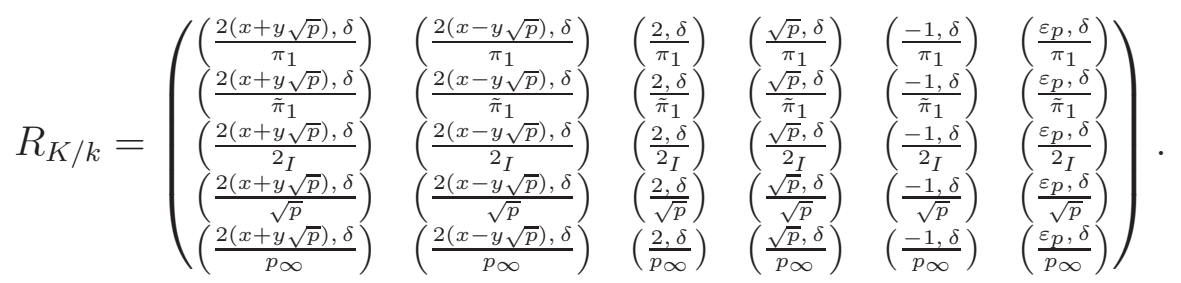

We consider the above matrix with coefficients in $\mathbb{F}_{2}$ by replacing 1 by 0 and -1 by 1 . Consequently, we can fill the Rédei matrix according to the values of the prime number $q$.

\begin{tabular}{|c|c|c|c|c|c|c|c|c|c|c|c|c|c|}
\hline - If & & & & & & 1 & - If & & & & $\frac{q}{p}$ & & $=-1$ \\
\hline \multirow{5}{*}{$R_{K / k}=$} & & 0 & & 0 & 0 & 0 & \multirow{5}{*}{$R_{K / k}=$} & 10 & 1 & 0 & 1 & 0 & \\
\hline & & 0 & & 0 & 0 & 0 & & 1 & 0 & 0 & 1 & 0 & 0 \\
\hline & 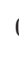 & 0 & & 1 & 1 & 0 & & c & 0 & 1 & 1 & 1 & 0 \\
\hline & & 0 & & 0 & 0 & 1 & & & 1 & 1 & 0 & 0 & 1 \\
\hline & ( & 0 & & 1 & 1 & 1 & & (c & 0 & 0 & 1 & 1 & 1 \\
\hline
\end{tabular}
Suppose that $q \equiv 1(\bmod 8)$, then $\left(\frac{2}{q}\right)=1$. 


\begin{tabular}{|c|c|c|c|c|c|c|c|c|c|c|c|c|c|}
\hline - If ( & & & & $\left.\frac{q}{p}\right)$ & & $=1$ & - If $\left(\frac{\mathrm{p}}{\mathrm{q}}\right.$ & & & & $\frac{q}{p}$ & & $=-1$ \\
\hline \multirow{5}{*}{$R_{K / k}=$} & & 0 & 0 & 0 & 0 & $1)$ & \multirow{5}{*}{$R_{K / k}=$} & & 1 & 0 & 1 & 0 & \\
\hline & ( & 0 & 0 & 0 & 0 & 1 & & & 0 & 0 & 1 & 0 & \\
\hline & & 1 & 1 & 1 & 1 & 0 & & & 1 & 1 & 1 & 1 & \\
\hline & & 1 & 1 & 0 & 0 & 1 & & & 0 & 1 & 0 & 0 & \\
\hline & ( & 0 & 0 & 1 & 1 & 1) & & ( & 0 & 0 & 1 & 1 & 1) \\
\hline
\end{tabular}

Consequently, $\operatorname{rank}\left(R_{K / k}\right)= \begin{cases}4, & \text { if }\left(\frac{p}{q}\right)_{4}=-1, \\ 3, & \text { if }\left(\frac{p}{q}\right)_{4}=-\left(\frac{q}{p}\right)_{4}=1, \\ 2, & \text { if }\left(\frac{p}{q}\right)_{4}=\left(\frac{q}{p}\right)_{4}=1 .\end{cases}$

Thus from the 4-rank formula of $C l(K)$ (see, $(2)$ ), we obtain

$$
r_{4}(K)= \begin{cases}0, & \text { if }\left(\frac{p}{q}\right)_{4}=-1, \\ 1, & \text { if }\left(\frac{p}{q}\right)_{4}=-\left(\frac{q}{p}\right)_{4}=1 \\ 2, & \text { if }\left(\frac{p}{q}\right)_{4}=\left(\frac{q}{p}\right)_{4}=1 .\end{cases}
$$

Suppose that $q \equiv 5(\bmod 8)$, then $\left(\frac{2}{q}\right)=-1$.

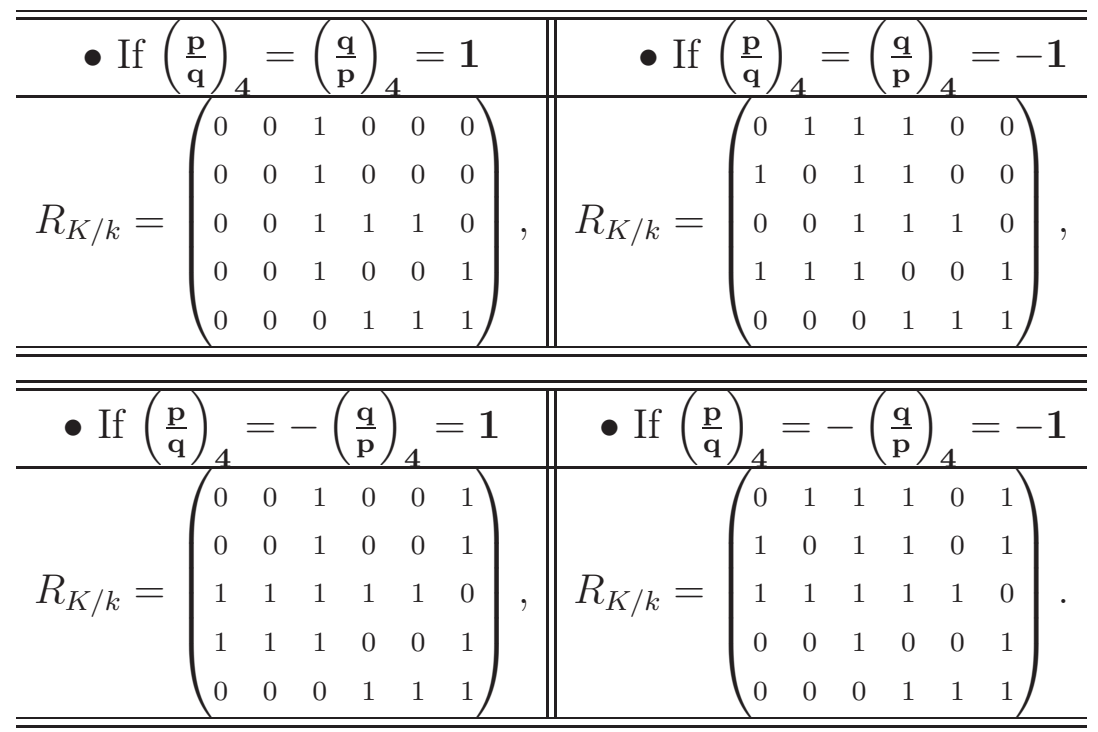

Consequently, $\operatorname{rank}\left(R_{K / k}\right)= \begin{cases}3, & \text { if }\left(\frac{p}{q}\right)_{4}=1 \\ 4, & \text { if }\left(\frac{p}{q}\right)_{4}=-1 .\end{cases}$

Then $r_{4}(K)= \begin{cases}1, & \text { if }\left(\frac{p}{q}\right)_{4}=1 \\ 0, & \text { if }\left(\frac{p}{q}\right)_{4}=-1\end{cases}$

Suppose that $q \equiv 3(\bmod 8)$, then $\left(\frac{2}{q}\right)=-1$.

If $\left(\frac{y}{q}\right)=1$ 


\begin{tabular}{c}
\hline • If $\left(\frac{\mathbf{q}}{\mathbf{p}}\right)_{4}=\mathbf{1}$ \\
$R_{K / k}=\left(\begin{array}{llllll}0 & 1 & 1 & 0 & 1 & 1 \\
1 & 1 & 1 & 1 & 1 & 0 \\
1 & 0 & 1 & 0 & 1 & 1 \\
0 & 0 & 1 & 0 & 0 & 1 \\
0 & 0 & 0 & 1 & 1 & 1\end{array}\right), \quad R_{K / k}=\left(\begin{array}{llllll}0 & 0 & 1 & 1 & 1 & 1 \\
0 & 1 & 1 & 0 & 1 & 0 \\
1 & 0 & 1 & 0 & 1 & 1 \\
1 & 1 & 1 & 0 & 0 & 1 \\
0 & 0 & 0 & 1 & 1 & 1\end{array}\right)$. \\
\hline
\end{tabular}

If $\left(\frac{y}{q}\right)=-1$

\begin{tabular}{c}
\hline \hline • If $\left(\frac{\mathbf{q}}{\mathbf{p}}\right)_{4}=\mathbf{1}$ \\
$R_{K / k}=\left(\begin{array}{cccccc}1 & 1 & 1 & 1 & 1 & 0 \\
1 & 0 & 1 & 0 & 1 & 1 \\
0 & 1 & 1 & 0 & 1 & 1 \\
0 & 0 & 1 & 0 & 0 & 1 \\
0 & 0 & 0 & 1 & 1 & 1\end{array}\right), \| R_{K / k}=\left(\begin{array}{llllll}1 & 0 & 1 & 0 & 1 & 0 \\
0 & 0 & 1 & 1 & 1 & 1 \\
0 & 1 & 1 & 0 & 1 & 1 \\
1 & 1 & 1 & 0 & 0 & 1 \\
0 & 0 & 0 & 1 & 1 & 1\end{array}\right)$. \\
\hline \hline
\end{tabular}

So $\operatorname{rank}\left(R_{K / k}\right)=4$ and thus $r_{4}(K)=0$.

Suppose that $q \equiv 7(\bmod 8)$, then $\left(\frac{2}{q}\right)=1$.

If $\left(\frac{y}{q}\right)=1$

\begin{tabular}{c}
\hline \hline • If $\left(\frac{\mathbf{q}}{\mathbf{p}}\right)_{4}=\mathbf{1}$ \\
$R_{K / k}=\left(\begin{array}{llllll}0 & 1 & 0 & 0 & 1 & 1 \\
1 & 1 & 0 & 1 & 1 & 0 \\
1 & 0 & 1 & 0 & 1 & 1 \\
0 & 0 & 1 & 0 & 0 & 1 \\
0 & 0 & 0 & 1 & 1 & 1\end{array}\right), \quad R_{K / k}=\left(\begin{array}{llllll}0 & 0 & 0 & 1 & 1 & 1 \\
0 & 1 & 0 & 0 & 1 & 0 \\
1 & 0 & 1 & 0 & 1 & 1 \\
1 & 1 & 1 & 0 & 0 & 1 \\
0 & 0 & 0 & 1 & 1 & 1\end{array}\right)$. \\
\hline \hline
\end{tabular}

If $\left(\frac{y}{q}\right)=-1$

\begin{tabular}{c}
\hline \hline - If $\left(\frac{\mathbf{q}}{\mathbf{p}}\right)_{4}=\mathbf{1}$ \\
$R_{K / k}=\left(\begin{array}{cccccc}1 & 1 & 0 & 1 & 1 & 0 \\
1 & 0 & 0 & 0 & 1 & 1 \\
0 & 1 & 1 & 0 & 1 & 1 \\
0 & 0 & 1 & 0 & 0 & 1 \\
0 & 0 & 0 & 1 & 1 & 1\end{array}\right), \quad R_{K / k}=\left(\begin{array}{llllll}1 & 0 & 0 & 0 & 1 & 0 \\
0 & 0 & 0 & 1 & 1 & 1 \\
0 & 1 & 1 & 0 & 1 & 1 \\
1 & 1 & 1 & 0 & 0 & 1 \\
0 & 0 & 0 & 1 & 1 & 1\end{array}\right)$. \\
\hline \hline
\end{tabular}

Consequently, $\operatorname{rank}\left(R_{K / k}\right)= \begin{cases}4, & \text { if }\left(\frac{q}{p}\right)_{4}=1, \\ 3, & \text { if }\left(\frac{q}{p}\right)_{4}=-1 .\end{cases}$

Then $r_{4}(K)= \begin{cases}0, & \text { if }\left(\frac{q}{p}\right)_{4}=1 \\ 1, & \text { if }\left(\frac{q}{p}\right)_{4}=-1\end{cases}$ 
Example 3.8. Keep previous hypotheses and notations. Here we give examples when $q \equiv 1$ $(\bmod 8)$.

(i) For $p=173$ and $q=41$, we have $173 \equiv 5(\bmod 8)$ and $\left(\frac{173}{41}\right)=1$. From Corollary $((2.2)-(\mathrm{a}))$, we obtain $r_{2}(K)=2$. Also $41 \equiv 1(\bmod 8)$ and $\left(\frac{173}{41}\right)_{4}=-1$, so the condition of Theorem ( (B)-(1)) are satisfied. Hence, $r_{4}(K)=0$, i.e., $C l_{2}(K) \cong$ $\mathbb{Z} / 2 \mathbb{Z} \times \mathbb{Z} / 2 \mathbb{Z}$

(ii) For $p=101$ and $q=17$, we have $101 \equiv 5(\bmod 8)$ and $\left(\frac{101}{17}\right)=1$. From Corollary $((2.2)$ (a)), we obtain $r_{2}(K)=2$. As $17 \equiv 1(\bmod 8)$ and $\left(\frac{101}{17}\right)_{4}=-\left(\frac{17}{101}\right)_{4}=1$, so the condition of Theorem ((B)-(1)) are satisfied. Hence, $r_{4}(K)=1$. In fact, by Pari/GP we get $C l_{2}(K) \cong \mathbb{Z} / 8 \mathbb{Z} \times \mathbb{Z} / 2 \mathbb{Z}$.

(iii) For $p=157$ and $q=17$, we have $157 \equiv 5(\bmod 8)$ and $\left(\frac{157}{17}\right)=1$. From Corollary $((2.2)$ $(\mathrm{a}))$, we obtain $r_{2}(K)=2$. Also $17 \equiv 1(\bmod 8)$ and $\left(\frac{157}{17}\right)_{4}=\left(\frac{17}{157}\right)_{4}=1$, so the condition of Theorem $((\mathrm{B})-(1))$ are satisfied. Hence, $r_{4}(K)=2$. Precisely, $C l_{2}(K) \cong \mathbb{Z} / 8 \mathbb{Z} \times \mathbb{Z} / 4 \mathbb{Z}$.

Example 3.9. Now an example when $q \equiv 3(\bmod 8)$.

For $p=53$ and $q=43$, we have $53 \equiv 5(\bmod 8)$ and $\left(\frac{53}{43}\right)=1$. From Corollary $((2.2)$ (a)), we obtain $r_{2}(K)=2$, and as $43 \equiv 3(\bmod 8)$, with the condition of Theorem $((\mathrm{B})-(2))$, hence $r_{4}(K)=0$. In other words, $C l_{2}(K) \cong \mathbb{Z} / 2 \mathbb{Z} \times \mathbb{Z} / 2 \mathbb{Z}$.

Example 3.10. The following two examples illustrate the 3rd case of Theorem B.

(i) For $p=269$ and $q=53$, we have $269 \equiv 5(\bmod 8)$ and $\left(\frac{269}{53}\right)=1$. The Corollary $((2.2)$ (a)) implies that, $r_{2}(K)=2$ and since the condition of Theorem ((B)-(3)) are satisfied, so, $r_{4}(K)=0$, i.e., $C l_{2}(K) \cong \mathbb{Z} / 2 \mathbb{Z} \times \mathbb{Z} / 2 \mathbb{Z}$.

(ii) For $p=293$ and $q=109$, we can easily verify that $293 \equiv 5(\bmod 8)$ and $\left(\frac{293}{109}\right)=1$, $109 \equiv 5(\bmod 8)$ and $\left(\frac{293}{109}\right)_{4}=1$, then the Theorem $((\mathrm{B})-(3))$ implies that $r_{4}(K)=1$, exactly, $C l_{2}(K) \cong \mathbb{Z} / 4 \mathbb{Z} \times \mathbb{Z} / 2 \mathbb{Z}$.

Example 3.11. We end with an example for the last case of Theorem B.

(i) For $p=173$ and $q=23$, we have $173 \equiv 5(\bmod 8)$ and $\left(\frac{173}{23}\right)=1,23 \equiv 7(\bmod 8)$ and $\left(\frac{23}{173}\right)_{4}=1$. According to Corollary $((2.2)-(\mathrm{a}))$ and Theorem $((\mathrm{B})-(4))$, we have $r_{4}(K)=0$.

(ii) Similarly for $p=149$ and $q=7$, we have $149 \equiv 5(\bmod 8),\left(\frac{149}{7}\right)=1,7 \equiv 7$ $(\bmod 8)$ and $\left(\frac{7}{149}\right)_{4}=-1$, we find that $r_{4}(K)=1$. By Pari/GP, we find $C l_{2}(K) \cong$ $\mathbb{Z} / 16 \mathbb{Z} \times \mathbb{Z} / 2 \mathbb{Z}$.

3.12. Case: $d=q_{1} q_{2}$ with $q_{1}, q_{2}$ are distinct prime numbers and $\left(\frac{p}{q_{1}}\right)=\left(\frac{p}{q_{2}}\right)=-1$. Let $p, q_{1}, q_{2}$ be different odd prime numbers such that $p \equiv 5(\bmod 8),\left(\frac{p}{q_{1}}\right)=\left(\frac{p}{q_{2}}\right)=-1$. Then the finite primes of $k$ ramifying in $K$, in this case, are: $\tilde{q}_{1}, \tilde{q}_{2}, 2_{I}$ and $(\sqrt{p})$, where $\tilde{q_{1}}=q_{1} O_{k}, \tilde{q_{2}}=q_{2} O_{k}, p O_{k}=(\sqrt{p})^{2}$ and $2_{I}=2 O_{k}$. To compute the 4-rank of the class group of $K$, by using the generalized Rédei-matrix, we need the following lemma:

Lemma 3.13. Keeping previous hypotheses and notations, then

a. For $i=1,2$ we have $\left(\frac{\varepsilon_{p}, \delta}{\tilde{q}_{i}}\right)=\left(\frac{-1}{q_{i}}\right)$ and $\left(\frac{2, \delta}{\tilde{q_{i}}}\right)=\left(\frac{-1, \delta}{\tilde{q}_{i}}\right)=1$. 
b. $\left(\frac{q_{i}, \delta}{\tilde{q}_{i}}\right)=-\left(\frac{-1}{q_{i}}\right)$ and $\left(\frac{q_{i}, \delta}{\tilde{q}_{j}}\right)=1$ for $i \neq j \in\{1,2\}$.

Proof. We have

a. $\quad\left(\frac{-1, \delta}{\tilde{q_{1}}}\right)=\left[\frac{-1}{\tilde{q_{1}}}\right]=\left(\frac{1}{q_{1}}\right)=1$.

- As $N_{k / \mathbb{Q}}\left(\varepsilon_{p}\right)=-1$, so by ([10, Ch $4 \S 4.1$ Proposition 4.2, p. 112]), $\left(\frac{\varepsilon_{p}, \delta}{\tilde{q_{1}}}\right)=\left[\frac{\varepsilon_{p}}{\tilde{q_{1}}}\right]=\left(\frac{N_{k / \mathbb{Q}}\left(\varepsilon_{p}\right)}{q_{1}}\right)=\left(\frac{-1}{q_{1}}\right)$, and $\left(\frac{\varepsilon_{p}, \delta}{q_{2}}\right)=\left(\frac{-1}{q_{2}}\right)$.

By the same argument, we get $\left(\frac{-1, \delta}{\tilde{q_{2}}}\right)=\left[\frac{-1}{\tilde{q_{2}}}\right]=\left(\frac{1}{q_{2}}\right)=1$.

- As $N_{k / \mathbb{Q}}(2)=4$, so by $\left(\left[10\right.\right.$, Ch $4 \S 4.1$ Proposition 4.2, p. 112]), $\left(\frac{2, \delta}{\tilde{q}_{1}}\right)=\left[\frac{2}{\tilde{q}_{1}}\right]=1$. Similarly, we have $\left(\frac{2, \delta}{\tilde{q_{2}}}\right)=1$.

b. $\left(\frac{q_{1}, \delta}{\tilde{q_{2}}}\right)=\left[\frac{q_{1}}{\tilde{q_{2}}}\right]=\left(\frac{q_{1}^{2}}{q_{2}}\right)=1$, and

$$
\begin{aligned}
\left(\frac{q_{1}, \delta}{\tilde{q_{1}}}\right) & =\left(\frac{q_{1},-q_{1}}{\tilde{q}_{1}}\right)\left(\frac{q_{1},-q_{2} \sqrt{p}}{\tilde{q_{1}}}\right)=\left(\frac{q_{1},-q_{2} \sqrt{p}}{\tilde{q_{1}}}\right)=\left[\frac{-q_{2} \sqrt{p}}{\tilde{q_{1}}}\right] \\
& =\left[\frac{-\sqrt{p}}{\tilde{q_{1}}}\right]=\left(\frac{-1}{q_{1}}\right)\left(\frac{p}{q_{1}}\right)=-\left(\frac{-1}{q_{1}}\right) .
\end{aligned}
$$

By the same argument, we get $\left(\frac{q_{2}, \delta}{\tilde{q_{1}}}\right)=1$ and $\left(\frac{q_{2}, \delta}{\tilde{q_{2}}}\right)=-\left(\frac{-1}{q_{2}}\right)$.

Remark 3.14. By similar calculations as in Lemma (3.2), we get

$$
\left(\frac{q_{1}, \delta}{\sqrt{p}}\right)=\left(\frac{q_{2}, \delta}{\sqrt{p}}\right)=-1 \text { and }\left(\frac{q_{1}, \delta}{p_{\infty}}\right)=\left(\frac{q_{2}, \delta}{p_{\infty}}\right)=1
$$

The symbols $\left(\frac{-, \delta}{2_{I}}\right)$ can be computed by using product formula except for $\left(\frac{\sqrt{p}, \delta}{\mathcal{P}_{i}}\right)$ which can be calculated by the formula $\left(\frac{\sqrt{p}, \delta}{\mathcal{P}_{i}}\right)=\left(\frac{-1, \delta}{\mathcal{P}_{i}}\right)\left(\frac{q_{1}, \delta}{\mathcal{P}_{i}}\right)\left(\frac{q_{2}, \delta}{\mathcal{P}_{i}}\right)$ consequence of $\left(\frac{-\delta, \delta}{\mathcal{P}_{i}}\right)=1$ for $\mathcal{P}_{i}=\tilde{q}_{1}, \tilde{q}_{2}, 2_{I},(\sqrt{p})$ or $p_{\infty}$.

If $d=q_{1} q_{2},\left(\frac{p}{q_{1}}\right)=\left(\frac{p}{q_{2}}\right)=-1$, then we can summarise the results in the following table:

\begin{tabular}{||c||c|c|c|c|c||}
\hline \hline element & $\tilde{q}_{1}$ & $\tilde{q}_{2}$ & $2_{I}$ & $(\sqrt{p})$ & $p_{\infty}$ \\
\hline$q_{1}$ & $-\left(\frac{-1}{q_{1}}\right)$ & 1 & $\left(\frac{-1}{q_{1}}\right)$ & -1 & 1 \\
\hline$q_{2}$ & 1 & $-\left(\frac{-1}{q_{2}}\right)$ & $\left(\frac{-1}{q_{2}}\right)$ & -1 & 1 \\
\hline 2 & 1 & 1 & -1 & -1 & 1 \\
\hline$\sqrt{p}$ & $-\left(\frac{-1}{q_{1}}\right)$ & $-\left(\frac{-1}{q_{2}}\right)$ & $-\left(\frac{-1}{d}\right)$ & 1 & -1 \\
\hline-1 & 1 & 1 & -1 & 1 & -1 \\
\hline$\varepsilon_{p}$ & $\left(\frac{-1}{q_{1}}\right)$ & $\left(\frac{-1}{q_{2}}\right)$ & $\left(\frac{-1}{d}\right)$ & -1 & -1 \\
\hline \hline
\end{tabular}

Proof of Theorem C. If $d=q_{1} q_{2}$ with $q_{1}, q_{2}$ are two different prime numbers such that $\left(\frac{p}{q_{1}}\right)=\left(\frac{p}{q_{2}}\right)=-1$, by corollary $(2.2)$ we have $r_{2}(K)=2$. As the finite primes of $k$ which ramify in $K$ are: $\tilde{q_{1}}, \tilde{q_{2}}, 2_{I},(\sqrt{p})$ where $\tilde{q_{1}}=q_{1} O_{k}, \tilde{q_{2}}=q_{2} O_{k}, 2_{I}=2 O_{k}$ and $(\sqrt{p})^{2}=p O_{k}$. So there exist prime ideals $\mathcal{H}_{1}, \mathcal{H}_{2}, \mathcal{H}_{3}, \mathcal{H}_{4}$ of $K$ such that

$$
\mathcal{H}_{1}^{2}=\tilde{q}_{1} O_{K}, \mathcal{H}_{2}^{2}=\tilde{q}_{2} O_{K}, \mathcal{H}_{3}^{2}=2_{I} O_{K}, \mathcal{H}_{4}^{2}=\sqrt{p} O_{K}
$$


Thus

- $\mathcal{H}_{1} \sigma\left(\mathcal{H}_{1}\right)=\mathcal{H}_{1} \mathcal{H}_{1} O_{K}=\tilde{q}_{1} O_{K}=q_{1} O_{K}$,

- $\mathcal{H}_{2} \sigma\left(\mathcal{H}_{2}\right)=\mathcal{H}_{2} \mathcal{H}_{2} O_{K}=\tilde{q}_{2} O_{K}=q_{2} O_{K}$,

- $\mathcal{H}_{3} \sigma\left(\mathcal{H}_{3}\right)=\mathcal{H}_{3} \mathcal{H}_{3} O_{K}=2{ }_{I} O_{K}=2 O_{K}$,

- $\mathcal{H}_{4} \sigma\left(\mathcal{H}_{4}\right)=\mathcal{H}_{4} \mathcal{H}_{4} O_{K}=\sqrt{p} O_{K}=\sqrt{p} O_{K}$.

where $\sigma$ is the generateur of the Galois group of $K$ over $k$.

Moreover, $E_{k}=\left\langle-1, \varepsilon_{p}\right\rangle$ and $-1, \varepsilon_{p},-\varepsilon_{p} \notin E_{k} \cap N_{K / k}(K)$ (Lemma 2.1). Then the $5 \times(4+2)$ generalized Rédei's matrix $R_{K / k}$ is

$$
R_{K / k}=\left(\begin{array}{llllll}
\left(\frac{q_{1}, \delta}{\tilde{q}_{1}}\right) & \left(\frac{q_{2}, \delta}{\tilde{q}_{1}}\right) & \left(\frac{2, \delta}{\tilde{q}_{1}}\right) & \left(\frac{\sqrt{p}, \delta}{\tilde{q}_{1}}\right) & \left(\frac{-1, \delta}{\tilde{q}_{1}}\right) & \left(\frac{\varepsilon_{p}, \delta}{\tilde{q}_{1}}\right) \\
\left(\frac{q_{1}, \delta}{\tilde{q}_{2}}\right) & \left(\frac{q_{2}, \delta}{\tilde{q}_{2}}\right) & \left(\frac{2, \delta}{\tilde{q}_{2}}\right) & \left(\frac{\sqrt{p}, \delta}{\tilde{q}_{2}}\right) & \left(\frac{-1, \delta}{\tilde{q}_{2}}\right) & \left(\frac{\varepsilon_{p}, \delta}{\tilde{q}_{2}}\right) \\
\left(\frac{q_{1}, \delta}{2}\right) & \left(\frac{q_{2}, \delta}{2} I_{2}\right. & \left(\frac{2, \delta}{2}\right) & \left(\frac{\sqrt{p}, \delta}{2}\right) & \left(\frac{-1, \delta}{2_{1}}\right) & \left(\frac{\varepsilon_{p}, \delta}{2_{I}}\right) \\
\left(\frac{q_{1}, \delta}{\sqrt{p}}\right) & \left(\frac{q_{2}, \delta}{\sqrt{p}}\right) & \left(\frac{2, \delta}{\sqrt{p}}\right) & \left(\frac{\sqrt{p}, \delta}{\sqrt{p}}\right) & \left(\frac{-1, \delta}{\sqrt{p}}\right) & \left(\frac{\varepsilon_{p}, \delta}{\sqrt{p}}\right) \\
\left(\frac{q_{1}, \delta}{p_{\infty}}\right) & \left(\frac{q_{2}, \delta}{p_{\infty}}\right) & \left(\frac{2, \delta}{p_{\infty}}\right) & \left(\frac{\sqrt{p}, \delta}{p_{\infty}}\right) & \left(\frac{-1, \delta}{p_{\infty}}\right) & \left(\frac{\varepsilon_{p}, \delta}{p_{\infty}}\right)
\end{array}\right) .
$$

We consider the above matrix with coefficients in $\mathbb{F}_{2}$ by replacing 1 by 0 and -1 by 1 .

\begin{tabular}{|c|c|c|c|c|c|c|c|c|c|c|c|c|c|}
\hline - If $\left(q_{1}, q\right.$ & & & , & ) & $(\mathrm{m}$ & $\mathrm{d}$ & - If $\left(q_{1}, q\right.$ & & ( & 3 & (1 & 100 & \\
\hline \multirow{5}{*}{$R_{K / k}=$} & 1 & 0 & 0 & 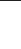 & ( & & \multirow{5}{*}{$R_{K / k}=$} & & 0 & 0 & 1 & 0 & \\
\hline & & 1 & 0 & & 0 & & & & 0 & 0 & 0 & 0 & 1 \\
\hline & ( & 0 & 1 & & & & & & 1 & 1 & 0 & 1 & 1 \\
\hline & & 1 & 1 & & ) & & & & 1 & 1 & 0 & 0 & \\
\hline & ( & 0 & 0 & & & 1 & & ( & 0 & 0 & 1 & 1 & 1) \\
\hline
\end{tabular}

and

\begin{tabular}{|c|c|c|c|c|c|c|c|c|c|c|c|c|}
\hline - If $\left(q_{1}, q\right.$ & 2) & 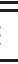 & $\overline{, 11}$ & & $\overline{\mathrm{mc}}$ & $\mathrm{d} 4$ & - - If $\left(q_{1}\right.$, & $E$ & 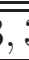 & ) & & $\mathrm{d}$ \\
\hline \multirow{5}{*}{$R_{K / k}=$} & $c$ & 0 & 0 & 0 & 0 & & \multirow{5}{*}{$R_{K / k}=$} & 0 & 0 & 0 & 0 & \\
\hline & 0 & 1 & 0 & 1 & 0 & 0 & & 0 & 0 & 0 & 0 & 1 \\
\hline & 1 & c & 1 & 0 & 1 & 1 & & 1 & 1 & 1 & 1 & \\
\hline & 1 & 1 & 1 & 0 & 0 & & & 1 & 1 & 0 & 0 & \\
\hline & 0 & ( & 0 & 1 & 1 & 1) & & 0 & 0 & 1 & 1 & 1) \\
\hline
\end{tabular}

So

$$
\operatorname{rank}\left(R_{K / k}\right)= \begin{cases}3, & \text { if }\left(q_{1}, q_{2}\right) \equiv(3,3) \quad(\bmod 4) \\ 4, & \text { otherwise }\end{cases}
$$

Then

$$
r_{4}(K)= \begin{cases}1, & \text { if }\left(q_{1}, q_{2}\right) \equiv(3,3) \quad(\bmod 4) \\ 0, & \text { otherwise }\end{cases}
$$

Example 3.15. We finish this work by illustrating the last Theorem with some examples:

(i) For $p=5, q_{1}=3$ and $q_{2}=7$, we have $p=5 \equiv 5(\bmod 8)$ and $\left(\frac{5}{3}\right)=\left(\frac{5}{7}\right)=-1$. From Corollary $((2.2)-(\mathrm{b}))$, we obtain $r_{2}(K)=2$. As $\left(q_{1}, q_{2}\right)=(3,7) \equiv(3,3)(\bmod 4)$, then, $r_{4}(K)=1$. In fact, $C l_{2}(K) \cong \mathbb{Z} / 4 \mathbb{Z} \times \mathbb{Z} / 2 \mathbb{Z}$. 
(ii) Likewise if $p=13, q_{1}=19$ and $q_{2}=83$, we obtain $r_{2}(K)=2$ and $r_{4}(K)=1$, but this time, $C l_{2}(K) \cong \mathbb{Z} / 16 \mathbb{Z} \times \mathbb{Z} / 2 \mathbb{Z}$.

For the case $\left(q_{1}, q_{2}\right) \not \equiv(3,3)(\bmod 4)$, we have the following examples:

(iii) Take $p=37, q_{1}=17$ and $q_{2}=29$, we have $p=37 \equiv 5(\bmod 8)$ and $\left(\frac{37}{17}\right)=\left(\frac{37}{29}\right)=$ -1. From Corollary $((2.2)-(\mathrm{b}))$, we obtain $r_{2}(K)=2$. Also $\left(q_{1}, q_{2}\right)=(17,29) \equiv(1,1)$ $(\bmod 4)$, so the condition of Theorem $(\mathrm{C})$ are satisfied. Hence, $r_{4}(K)=0$ and $C l_{2}(K) \cong \mathbb{Z} / 2 \mathbb{Z} \times \mathbb{Z} / 2 \mathbb{Z}$.

(iv) For $p=37, q_{1}=17, q_{2}=31$, we have $p=37 \equiv 5(\bmod 8),\left(\frac{37}{17}\right)=\left(\frac{37}{31}\right)=-1$ and $\left(q_{1}, q_{2}\right)=(17,31) \equiv(1,3)(\bmod 4)$. we also find $C l_{2}(K) \cong \mathbb{Z} / 2 \mathbb{Z} \times \mathbb{Z} / 2 \mathbb{Z}$.

\section{ACKNOWLEDGMENT}

We would like to thank the unknown referee for his/her several helpful suggestions and for calling our attention to the missing details.

\section{REFERENCES}

[1] A. Azizi et M. Taous, Détermination des corps $k=\mathbb{Q}(\sqrt{d}, i)$ dont les 2-groupes de classes sont de type $(2,4)$ ou $(2,2,2)$, Rend. Istit. Mat. Univ. Trieste. 40, (2008), 93-116.

[2] A. Azizi et A. Mouhib, Sur le rang du 2-groupe de classes de $\mathbb{Q}(\sqrt{m}, \sqrt{d})$ oú $m=2$ ou un premier $p \equiv 1$ (mod 4), Trans. Amer. Math. Soc. 353, No 7, (2001), 2741-2752.

[3] A. Azizi et A. Mouhib, Le 2-rang du groupe de classes de certains corps biquadratiques et applications, Internat. J. Math. 15, No. 02, (2004), 169-182.

[4] A. Azizi, M. Taous and A. Zekhnini, On the unit index of some real biquadratic number fields, Turk. J. Math. (2018), no. 42, 703-715.

[5] E. Brown and Ch. J. Parry, The 2-class group of certain biquadratic number fields, J. reine angew. Math. 295, (1977), 61-71.

[6] E. Brown and Ch. J. Parry, The 2-class group of certain biquadratic number fields II, Pacific J. Math. 78, No. 1, (1978), 61-71.

[7] C. Chevalley, Sur la théorie du corps de classes dans les corps finis et les corps locaux, J. Fac. Sc. Tokyo, Sect. 1, t. 2, (1933), 365-476.

[8] G. Gras, Class field theory, from theory to practice, Springer Verlag 2003.

[9] J. A. Hymo and C. J. Parry, On relative integral bases for pure quartic fields, Indian J. Pure Appl. Math. 23, 1992, 359-376.

[10] F. Lemmermeyer, Reciprocity Laws. From Euler to Eisenstein, Springer Monographs in Math. (2000).

[11] C. J. Parry, Pure quartic number fields whose class numbers are even, J. Reine Angew. Math. 264(1975), 102-112.

[12] C. J. Parry, A genus theory for quartic fields, J. Reine Angew. Math. 314 (1980), 40-71.

[13] The PARI Group, PARI/GP version 2-11-3, Univ. Bordeaux, 2020, http: //pari.math.u-bordeaux.fr.

[14] Y. Qin, The generalized Rédei-matrix, Math. Z. 261 (2009), 23-37.

[15] M. Taous, Capitulation des 2 -classes d'idéaux de certains corps $\mathbb{Q}(\sqrt{d}, i)$ de type $(2,4)$, thèse, Université. Mohammed Premier Faculté des Science, Oujda. 2008.

[16] H. C. Williams, The quadratic character of a certain quadratic surds, Utilitas Math. 5, (1974), 49-55.

Moulay Ismail University of Meknes. Faculty of Sciences and Technology, P.O. Box 509-Boutalamine, 52000 Errachidia

E-mail address: haynou_mbarek@hotmail.com

E-mail address: taousm@hotmail.com 